\title{
EVALUATION OF OPTICAL CHARACTERISTICS BASED UPON STRUCTURAL PARAMETERS OF PULP SHEET
}

\author{
By Makio Hasuike, Takashi Kawasaki and Koji Murakami \\ (Faculty of Agriculture, Kyoto University, \\ Sakyo-ku, Kyoto, 606, Japan)
}

\begin{abstract}
Interrelationships between optical properties and layered structure of pulp sheets were discussed quantitatively by applying the layered model theory developed by Scallan and Borch.

The number of optically-effective layers in the sheet, determined from optical data was a good structural parameter which relates optical properties to the layered structure of the sheet. For the sheets from pulps of different grade of bleaching, the values of the number of layers conveniently estimated from their specific scattering coefficient and basis weight coincided with those obtained from stepwise computer calculation by applying the layered model theory.

It is found also that the number of effective layers for diffusely incident light is practically affected by two geometrical parameters of the sheet, the number of fibrous layers in the $z$-direction and the degree of orientation of fibrous layers to the plane of the sheet.

Fairly good agreement in the number of optically-effective layers estimated from optical data and from cross sections of the sheet was obtained experimentally.
\end{abstract}

\section{Introduction}

A description of the optical characteristics of paper in terms of structure and fiber properties is important to establish the optimum use of a broader range of wood and non-wood fibers for papermaking.

As a conventional method to evaluate the opacifying quality of pulp and paper, the specific scattering coefficient is determined experimentally by applying the Kubelka-Munk theory. However, the real structure of paper is very different from the homogeneous model assumed in the K-M theory. Based on this fact, Scallan and Borch ${ }^{1}$ ) described the optical properties of paper in terms of a layer model assuming the sheet to be a pile of parallel layers of alternating air and solid material. This is more closely related to real paper structure. Therefore, the optical properties will be described in terms of the structural parameters of the sheet by applying the Scallan-Borch layered model theory.

In this paper, an attempt is made to clarify the optical scattering process in a paper sheet in terms of the structural parameters of the sheet. To estimate the structural parameters from optical properties, the applicability of reflectance theories to the optical data on the sheets from pulps of different degrees of bleaching was examined. -Furthermore, the structural parameters determined from the optical data were discussed based upon the geometrical structure of cross sections of the sheets.

\section{Experimental}

\subsection{Sample pulp sheets}

A series of fully- and semi-bleached softwood kraft pulps from Akamatsu (Pinus densiflora Sieb. and Zucc.) was prepared by three stage bleaching sequence, chlorination - alkaline extraction - hypochlorite bleaching, for the evaluation of structural parameters determined from the optical data by applying the Scallan-Borch theory. (The pulp was treated with different amount of hypochlorite.)

In addition to the Akamatsu pulp, unbleached hardwood kraft pulps of Bagtikan (Parashorea 
plicata Blco.) and Narig (Vatica mangachapoi Brandis) were prepared to examine the relationship between the structural parameters obtained from optical data and those obtained from cross sections of the sheets.

Each pulp was put into a JIS type classifier with a 150 -mesh screen to obtain the fines-free pulp. Fifteen grams of pulp was beaten in a PFI mill at $10 \%$ consistency with $0.2 \mathrm{~mm}$ clearance for three levels of revolutions. To prepare the handsheets of less glossy surface, the wet handsheets having $60 \mathrm{~g} / \mathrm{m}^{2}$ in basis weight were pressed and dried between blotting papers. That is, specular reflection from the surface should be minimized for applying the K-M theory ${ }^{2)}$ and the S-B theory on the optical data.

\subsection{Measurement of optical properties}

To estimate the sheet reflectance $\left(R_{0}\right)$ and multiple sheet reflectance $\left(R_{\infty}\right)^{\dagger 1}$, reflectance spectra of a single handsheet and a pile of the sheets in which no light is transmitted, were measured, respectively, in the wave length range of 400-800 $\mathrm{nm}$ using a recording spectrophotometer with an integrating sphere, UV-365 of Shimadzu Co. In this instrument, parallel incident light falls on a sample sheet of $24 \times 8 \mathrm{~mm}$ size at an angle of $7^{\circ}$, and the reflectance over a hemisphere is detected. Barium sulfate, DIN-Standard 5033, was used as a reference for a white standard.

\subsection{Microscopic analysis of layered structure of the sheets}

In order to analyze the layered structure of handsheets, cross sections of the sheets were prepared. Small pieces $(5 \times 12 \mathrm{~mm})$ of each sheet were embedded in epoxy resin (Epok 812) for sectioning. Sections of $1-2 \mu \mathrm{m}$ in thickness were cut on an ultramicrotome (Sorvall, JB-4). To avoid fracture in the sections during staining, unstained sections were examined and photographs were taken under the Nomarski differential interference microscope (Olympus Co.). The principal advantage of this method of microscopy ${ }^{3)}$ rather than phase-contrast microscopy is that a clear image of the unstained section may be obtained.

Several enlarged photographs at a magnification of approximately $500 \times$ were used for the estimation of the structural parameters. The number ${ }^{\mp 1}$ The common term "brightness" is applicable for $R_{\infty}$ measured at a wave length of $457 \mathrm{~nm}$. of fibrous layers in the $z$-direction was measured by the point counting method ${ }^{4}$ with a series of equidistant test lines normal to the center line of the sheet. The degree of orientation of crosssectional peripheries of fibrous layers to the plane of the sheet was also estimated from the frequencies of intersections of fibrous elements with unit length test lines on the sheet. The center line in the sheet was determined according to the procedure proposed by Crosby 5 ).

\section{Results and Discussion}

\subsection{Structural parameters from optical data of the sheet}

3.1.1 Structural parameters in reflectance theory

According to the Scallan-Borch layered model theory ${ }^{1)}$, the reflectance of a sheet $\left(R_{0}=R_{n_{o}}\right)$ that is composed uniformly of $n_{0}$ layers is given by

$$
R_{0}=R_{n_{o}}=R_{1}+R_{n_{o-1}} T_{1}^{2} /\left(1-R_{1} R_{n_{o-1}}\right)
$$

where $R_{1}$ and $T_{1}$ denote reflectance and transmittance of a sheet composed of one layer, respectively. $R_{n_{O}-1}$ is reflectance of a sheet of $n_{o}-1$ layers.

If absorption of light is negligible in the sheet, its reflectance $\left(R_{0}\right)$ is expressed by the reflectivity $(r)$ of pulp fibers and the number of opticallyeffective layers $\left(n_{o}\right)$ in the sheet.

$$
1 / R_{0}=1+[(1-r) / 2 r]\left(1 / n_{o}\right)
$$

Equation [2] implies the number of opticallyeffective layers in the sheet can be estimated from its reflectance, because reflectivity ${ }^{\dagger 2}$ is to be an optical constant intrinsic to the sheet material.

On the other hand, the relationship between the specific scattering coefficient $(S)$ and reflectivity $(r)$ of the sheet was derived by Tsuchida ${ }^{6)}$ and Kortïm ${ }^{7)}$, assuming that the absorption of light in pulp fibers is negligible.

$$
S=[2 r /(1-r)]\left(n_{o} / W\right)
$$

where $W$ is basis weight. Equation [3] also describes the dependence of the scattering coeffi-

$\uparrow^{2}$ Reflectivity is defined as the reflection factor of the solid-air interface, and should only depend on the refractive index of the solid material and the angle of incidence of the light. For a material with the refractive index of cellulose, $r$ is 0.10 for diffusely incident light. 
cient on the number of optically-effective layers in the sheet.

Since real sheets have a non-uniform distribution of the layers, the reflectance of such a sheet will vary from point to point in the sheet. As an approximation to the real sheet, Borch ${ }^{8)}$ and later the author ${ }^{9}$ introduced a Poisson distribution without bringing further variables into the equation system. That is, the reflectance of a sheet having $\bar{n}_{o}$ of mean number of layers was given by

$$
R_{0}=R_{\bar{n}_{0}}=\sum_{k=1}^{\infty} R_{k} \exp \left(-\bar{n}_{o}\right) \bar{n}_{o}^{-k} / k !
$$

where $R_{k}$ is reflectance of a sheet composed of $k$ layers.

The values of $n_{o}$ estimated from Eq. [2] and from Eq. [3] should be always smaller than $\bar{n}_{o}$ estimated from Eq. [4] by stepwise computer calculation. This is because $R_{0}$ does not vary linearly with $n_{0}$, and the decreased contribution to the number would be approximately compensated by a factor of 1.1 for the Poisson distribution.

\subsubsection{Applicability of the theory}

The applicability of the above two equations, Eq. [2] and Eq. [3] respectively, to the estimation of structural parameters from optical data was examined on sheets prepared from different grades of bleached pulps.

Figure 1 shows the plot of reciprocal reflectance

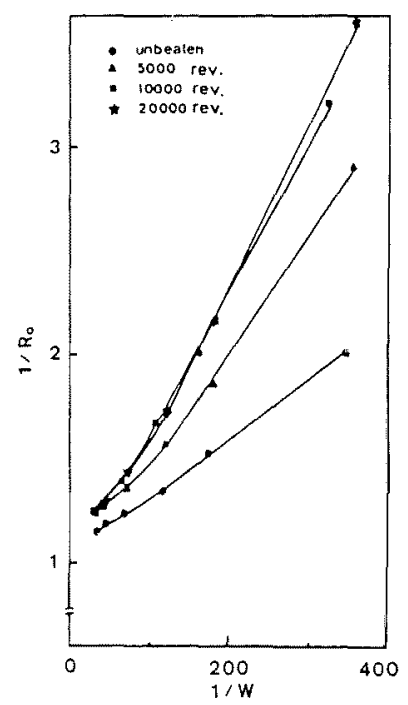

Fig. 1 Plot of reciprocal reflectance $\left(1 / R_{0}\right)$ versus reciprocal basis weight $(1 / W)$ for the sheets from fully-bleached Akamatsu pulp. $\left(1 / R_{0}\right)$ versus reciprocal basis weight $(1 / W)$ for the fully-bleached Akamatsu pulp sheets. Instead of the number of layers, basis weight of the sheet was used in this diagram, as indicated by Scallan and Borch ${ }^{1}$. These plots should fall on a straight line, as expected from Eq. [2]. As seen in Fig. 1, a linear relationship was maintained with relatively low basis weight levels. However, a slight deviation from the linear relationship was observed with an increase in basis weight, because the absorbancy of the sheet becomes greater to some extent. Therefore, the deviation would be significant for unbleached pulp sheets.

In this way, the influence of absorbancy of the sheet on the specific scattering coefficient was evaluated on the sheets prepared from Akamatsu pulp of different grade of bleaching.

Figure 2 shows the effects of beating and bleaching on the specific scattering and absorption coefficients ( $S$ and $K$ respectively) of the sheets. The variation of the scattering coefficient with beating was found to be almost independent of the degree of bleaching, and the slight differences within the identical level of beating would be attributed to differences in internal surface area due to differences in fiber stiffness of different bleached pulps. The absorption coefficient is almost constant with beating, which is dependent upon the chemical composition of the fibers. Therefore, the specific scattering coefficient would
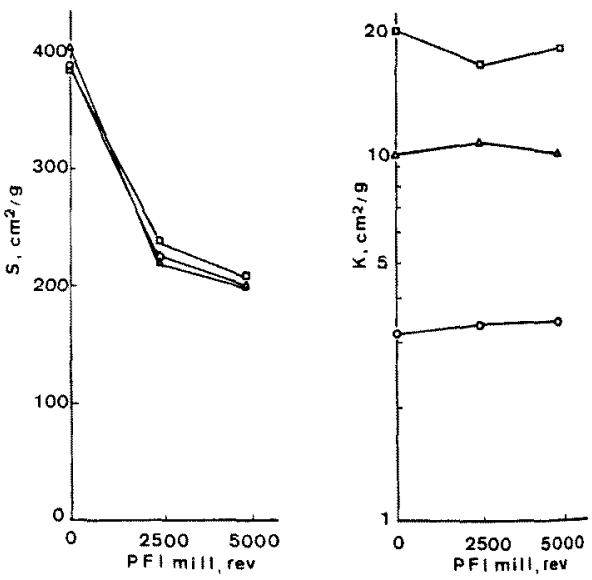

Fig. 2 Effects of beating and bleaching on the specific scattering and absorption coefflcients, $S$ and $K$ respectively, of the Akamatsu pulp sheets (Degree of bleaching; $O$ : high, $\Delta$ : medium and $\square$ : low). 
a)

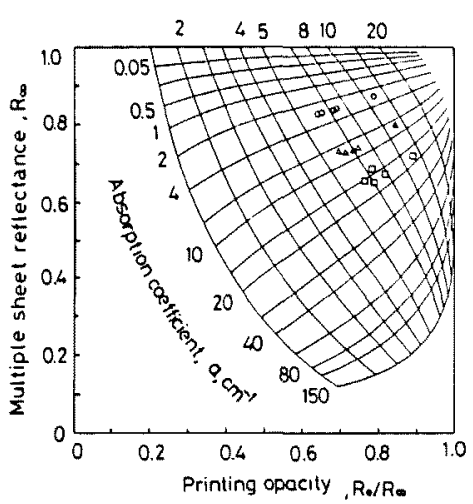

b) Number of optically-ellective layers

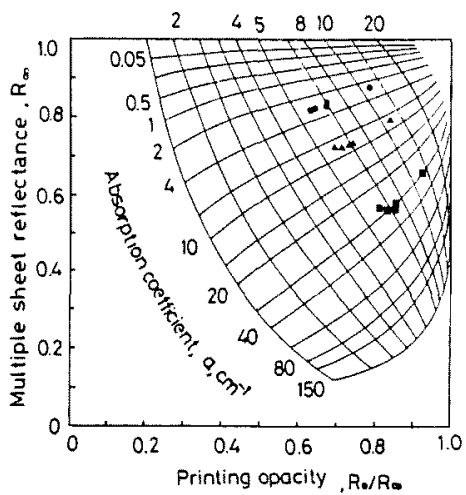

Fig. 3 Scallan-Borch plots of multiple sheet reflectance $\left(R_{\infty}\right)$ versus printing opacity $\left(R_{0} / R_{\infty}\right)$ for Akamatsu pulp sheets of $60 \mathrm{~g} / \mathrm{m}^{2}$ in basis weight.

Notes; a) Series of different bleached pulp sheets measured at $557 \mathrm{~nm}$ in wave length (Degree of bleaching; $O$ : high, $\triangle$ : medium and $\square$ : low), and b) Series of different wave lengths in measurement for medium bleached pulp sheets (Wave length; : 657 $\mathrm{nm}, \boldsymbol{\Delta}: 557 \mathrm{~nm}$ and $\mathbf{a}: 457 \mathrm{~nm}$ ).

be applicable for the estimation of the number of optically-effective layers in the sheet that multiple sheet reflectance $\left(R_{\infty}\right)$ is higher than 0.5 .

Figure 3 shows Scallan-Borch plots ${ }^{10}$ ) of multiple sheet reflectance $\left(R_{\infty}\right)$ versus printing opacity $\left(R_{0} / R_{\infty}\right)$ for Akamatsu pulp sheets of $60 \mathrm{~g} / \mathrm{m}^{2}$ in basis weight. The data are superimposed on the theoretical curves calculated using Eq. [4] for Poisson distribution of the number of layers and $r=0.1$. In Fig. 3 , the experimental beating plots for three different bleached pulps and for three different wave lengths in measurement follow the theoretical curves of constant absorption coefficient $(a)$. At the same levels of beating, plots are almost on the curves of a constant number of layers in the sheets prepared from pulps of different bleaching degrees.

Figure 4 shows the effect of light absorptive properties of the sheets on the number of layers estimated from optical data. The variation index in Fig. 4 indicates the ratio of the number of layers estimated by Eq. [2] and by Eq. [3] to the mean number of layers $\left(\bar{n}_{o}\right)$ calculated from experimental values of $R_{0}$ and $R_{\infty}$. Practically, the value of $\bar{n}_{o}$ necessary for $R_{0}$ is determined by stepwise computer calculation using Eq. [4]. With an increase in absorption coefficient of the sheet, deviation from unity was observed in the number estimated by Eq. [2]. Nevertheless, unity was maintained in the number estimated by Eq. [3]. It is noteworthy that there is good agreement

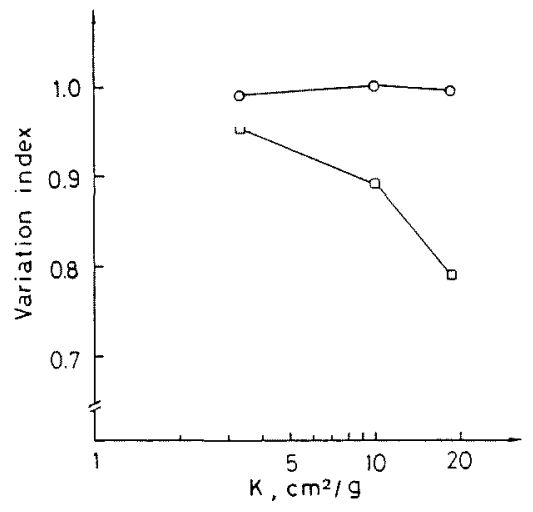

Fig. 4 Effect of light absorptive properties of the sheets on the number of optically-effective layers estimated from their optical data (口: number of layers from Eq. [2], and $O$ : number of layers from Eq. [3]).

Notes; Variation index indicates the ratio of the number from Eq. [2] and from Eq. [3] to that estimated by the mean number of layers necessary for the calculation of a reflectance which is equivalent to the measured one. 
between the values estimated from continuous and discontinuous model theories.

\subsection{Structural parameters from cross section of the sheet}

Scallan and Borch ${ }^{1)}$ estimated the number of layers from the specific surface area measured by nitrogen absorption. However, the number of layers could also be estimated directly from geometrical analysis on the cross sections of the sheets. Therefore, the fundamental role of layered structure on the optical properties would be revealed by clarifying the relationship between structural parameters obtained from optical data and from cross sections of the sheets.

Layered structure of paper sheet is affected not only by beating and wet pressing during the sheet forming process but also by wall thickness of pulp fibers in the sheet.

Figure 5 shows layered models for thin- and thick-walled fiber sheets (a-1 and a-2) and their polar diagrams of the number of intersections of fibrous elements with the test lines (b-1 and $b-2)^{11}$ ) The differences in surface irregularities, which are not taken into account in the reflectance theory, are remarkable between thin- and thick-walled fiber sheets. For diffusely incident light, not only the fibrous layers in the z-direction but also the layers in the other directions will affect the reflection and transmission of light in the sheet. The layered structure of the thin-walled fiber sheet is analogous to that of the Scallan-Borch layered

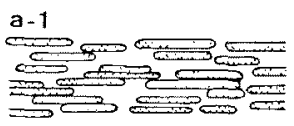

b- 1

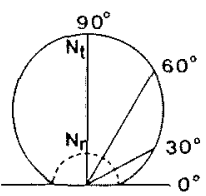

a- 2

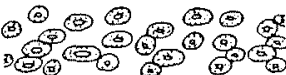

b- 2

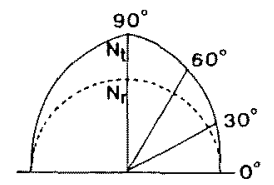

Fig. 5 Layered models for thin- and thick-walled fiber sheets (a), and their polar diagrams of the number of fibrous elements intersected by unit length test lines $(b)^{11)}$.

Note; $N_{r}$ indicates the number of randomlyoriented fibrous elements and $N_{t}$ includes the number of randomly-oriented and parallel fibrous elements to the plane of the sheet. model and the number of effective layers for diffused light will be as many as the number of fibrous layers in the $z$-direction of the sheet. On the other hand, the number of effective layers is greater than the number of fibrous layers in the z-direction in thick-walled fiber sheet for diffusely incident light, as the results of deviation from Scallan-Borch layered model.

This consideration will lead to the conclusion that the degree of orientation of fibrous layers of the sheet should be taken into account for the estimation of optical properties from structural parameters of the sheet. The degree of orientation of cross-sectional peripheries of fibrous layers to the plane of the sheet $(\varepsilon)$ was determined from the number of intersections of fibrous elements with unit length test lines, $N_{t}$ and $N_{r}{ }^{4}$ ).

$$
\varepsilon=\left(N_{t}-N_{r}\right) /\left[N_{t}+(\pi / 2-1) N_{r}\right]
$$

where $N_{t}$ is the number of intersected fibrous elements with test lines normal to the center line of the sheet, and $N_{r}$ is the number intersected with test lines parallel to the center line. $N_{t}$ indicates the total number of fibrous elements, i.e., those oriented randomly as well as those parallel to the plane of the sheet, and $N_{r}$ indicates only the number of randomly-oriented fibrous elements, respectively. Then, the anisotropy parameter $\left(E^{*}\right)$ which indicates the contribution of orientation of fibrous layers to the reflection of diffusely incident light was estimated.

$$
E^{*}=[\varepsilon(2-\pi)+\pi] / 2
$$

$E^{*}$ has a value of approximately 1.0 for a pile of thin filmy layer materials such as ideal layered model of Scallan and Borch, and 1.57 for an agglomerate of cubic particles.

Figure 6 shows an example of cross sections of the sheets from Akamatsu pulp. The results of the geometrical analysis on the cross sections are summarized in Table 1. The values of the anisotropy parameter $\left(E^{*}\right)$ for Narig pulp sheets were greater than those for Akamatsu and Bagtikan pulp sheets, because the layered structure of Narig sheets had a comparatively low degree of orientation of cross-sectional peripheries of the fibers ( $\varepsilon$ ) in the sheets. Furthermore, the anisotropy of the sheet decreased with the increase in degree of beating. For pulp sheets used here, the values 


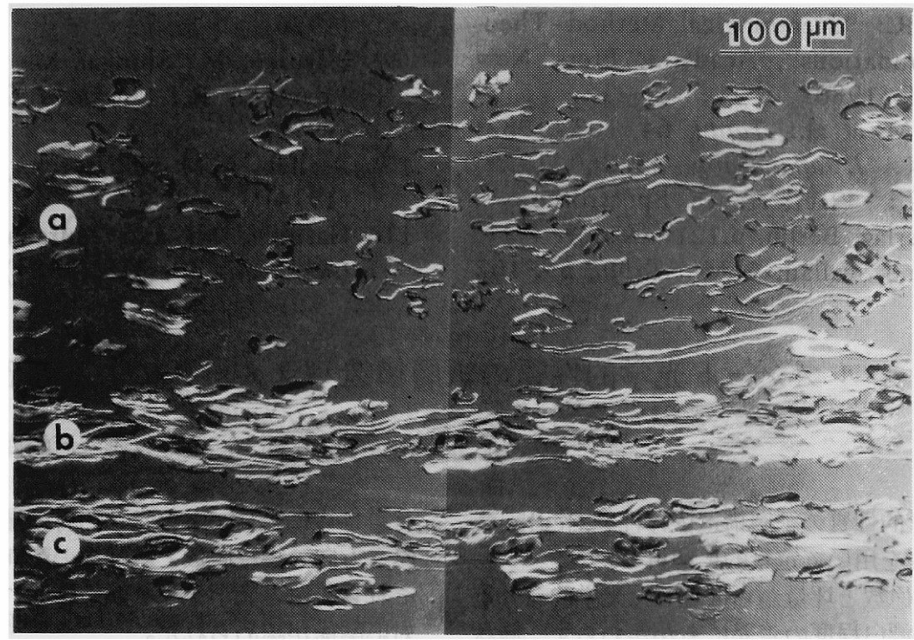

Fig. 6 Layered structure of the sheets prepared from Akamatsu pulp (PFI mill beating; a) 0 rev., b) 2500 rev. and c) 5000 rev.).

Table 1 Structural parameters estimated from geometrical and optical data for the unbleached pulp sheets.

\begin{tabular}{|c|c|c|c|c|c|c|c|c|}
\hline \multirow{2}{*}{$\begin{array}{l}\text { Sample } \\
\text { pulp }\end{array}$} & \multirow{2}{*}{$\begin{array}{l}\text { PFI mill } \\
\text { (rev.) }\end{array}$} & \multirow{2}{*}{$\begin{array}{c}\text { Basis weight } \\
\left(\mathrm{g} / \mathrm{m}^{2}\right)\end{array}$} & \multicolumn{2}{|c|}{ Number of fibrous layers } & \multicolumn{2}{|c|}{ Structural anisotropy } & \multicolumn{2}{|c|}{ Optically-effective layer } \\
\hline & & & $\bar{n}_{f}{ }^{a)}$ & $\sigma_{n_{f}}{ }^{\mathrm{b})}$ & ${ }^{c)}$ & $\left.E^{*} \mathrm{~d}\right)$ & $n_{g}{ }^{\mathrm{e})}$ & $\left.\bar{n}_{o} \mathrm{f}\right)$ \\
\hline \multirow[t]{3}{*}{ Akamatsu } & 0 & 59.8 & 9.7 & 3.0 & 0.562 & 1.25 & 12.2 & 11.5 \\
\hline & 2500 & 60.4 & 5.3 & 1.9 & 0.720 & 1.16 & 6.1 & 7.1 \\
\hline & 5000 & 61.0 & 5.4 & 2.7 & 0.702 & 1.17 & 6.7 & 6.0 \\
\hline \multirow[t]{3}{*}{ Bagtikan } & 0 & 60.9 & 10.6 & 2.6 & 0.683 & 1.18 & 12.5 & 11.8 \\
\hline & 5000 & 56.7 & 8.4 & 1.5 & 0.733 & 1.15 & 9.7 & 9.1 \\
\hline & 20000 & 57.2 & 7.7 & 1.3 & 0.728 & 1.15 & 8.9 & 8.1 \\
\hline \multirow[t]{3}{*}{ Narig } & 0 & 60.1 & 8.6 & 2.4 & 0.415 & 1.33 & 11.4 & 11.4 \\
\hline & 5000 & 59.4 & 8.2 & 1.7 & 0.504 & 1.28 & 10.5 & 10.3 \\
\hline & 20000 & 57.2 & 7.9 & 1.5 & 0.541 & 1.26 & 10.0 & 10.7 \\
\hline
\end{tabular}

a) $\bar{n}_{f}$ : mean number of fibrous layers, b) $\sigma_{n_{f}}:$ standard deviation of the number of fibrous layers, c) $\varepsilon:$ degree in orientation of cross-sectional peripheries of fibrous layers to the plane of the sheet, ${ }^{\text {d) }} E^{*}$. anisotropy parameter of fibrous layers given by $E^{*}=[\varepsilon(2-\pi)+\pi] / 2$, e) $n_{g}$ : number of optically-effective layers estimated by the product of $\bar{n}_{f}$ and $E^{*}$, f) $\bar{n}_{o}$ : mean number of optically-effective layers necessary for the calculation of a reflectance which is equivalent to the measured one.

of the number of effective layers for diffusely incident light $\left(n_{g}\right)$ were estimated to be approximately $1.15-1.33$ times greater than those of fibrous layers in the z-direction of the sheets $\left(\bar{n}_{f}\right)$. The last column in the Table represents the mean number of optically-effective layers $\left(\bar{n}_{o}\right)$ necessary for the calculation of a reflectance which is equivalent to the measured one. Fairly good agreement between the values of the number of optically-effective layers estimated from cross sections and from optical data was found.

Consequently, the variations in optical properties with the changes in sheet structure would be interpreted in terms of the "number of opticallyeffective layers" which is obtainable both from optical properties and from geometrical structure of the sheet.

\section{References}

1) Scallan, A. M. and Borch, J.: Tappi, 55, 583 (1972)

2) Stenius, A. S.. Svensk Papperstidn., 56, 607 (1953)

3) Allen, R. D., David, G. B. and Nomarski, G.: Z. wiss. Mikr., 69, 193 (1969) 
4) Weibel, E. R.: "Stereological Method-Theoretical Foundations", Academic Press, New York, p.305 (1980)

5) Crosby, C. M. et al.: Tappi, 64, 103 (1982)

6) Tsuchida, K.: J. Japan Tappi, 33, 260 (1979)

7) Kortüm, G.: "Reflectance Spectroscopy", Springer Verlag, Berlin, p.121 (1969)

8) Borch, J. and Scallan, A. M.: Tappi, 59, 102
(1976)

9) Hasuike, M., Shingai, M., Murakami, K. and Imamura, R.: Mokuzai Gakkaishi, 25, 132 (1979)

10) Scallan, A. M. and Borch, J.: Tappi, 57, 143 (1974)

11) Hasuike, M. and Murakami, K.: Mokuzai Gakkaishi, 30, 315 (1984)

\title{
パルプシート光学的性質の紙層構造パラメータによる評価
}

\author{
京都大学農学部蓮池牧雄, 河崎尚志, 村上浩二 \\ パルブシート光学的性質の紙層構造依存性を,Scallan- \\ Borchの層状理論を用いて定量的に解析した。 \\ シ一トの比散乱係数と坪量加ら推定した光学的有効層 \\ の数は、パルプの漂白程度の差異にあかかわらず，層状 \\ 理論を用いて推定した值にはぼ一致し，光学的性質に関 \\ 与寸る紙層構造パラメータとして用い得ることが判明し \\ た。さらに、シートの光学的有奻層の数をシート断面の

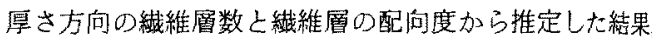 \\ それらの梿々光学データから得られた值との間に良好な \\ 対応関係が認めら扎た。 \\ したがって，シートの構造と光学的性質を光学的有效 \\ 層数を用いて定量的に対応つけ得るこよが判明した。
}

